\title{
Hypersphere World-Universe Model. Tribute to Classical Physics
}

\author{
Vladimir S. Netchitailo \\ Biolase Inc., Irvine, CA, USA \\ Email: v.netchitailo@sbcglobal.net
}

How to cite this paper: Netchitailo, V.S. (2018) Hypersphere World-Universe Model. Tribute to Classical Physics. Journal of High Energy Physics, Gravitation and Cosmology, 4, 441-470.

https://doi.org/10.4236/jhepgc.2018.43024

Received: April 24, 2018

Accepted: June 26, 2018

Published: June 29, 2018

Copyright $\odot 2018$ by author and Scientific Research Publishing Inc. This work is licensed under the Creative Commons Attribution International License (CC BY 4.0).

http://creativecommons.org/licenses/by/4.0/

(c) (i) Open Access

\begin{abstract}
This manuscript summarizes the results of Classical Physics before Quantum Mechanics and Hypotheses proposed by classical physicists from the 17th until the beginning of 21 st century. We then proceed to unify these results into a single coherent picture in frames of the developed Hypersphere World-Universe Model (WUM). The Model proposes 5 types of Dark Matter particles and predicts their masses; models the origin, evolution, and structure of the World and Macroobjects; provides a mathematical framework that ties together a number of Fundamental constants and allows for direct calculation of their values.
\end{abstract}

\section{Keywords}

Classical Physics, Hypersphere World-Universe Model, Medium of the World, Dark Matter Particles, Gravitoelectromagnetism, Cosmic Neutrino Background, Macroobjects Structure, Emergent Phenomena, Q-Dependent Cosmological Parameters

\section{Introduction}

This manuscript concludes the series of papers [1]-[9] published by "Journal of High Energy Physics, Gravitation and Cosmology" journal. Many results obtained there are quoted in the current work without a full justification; an interested reader is encouraged to view the referenced papers in such cases. The article does not provide an overview of Hypersphere World-Universe Model (WUM), so please refer to manuscripts for that.

In this paper, we show that WUM is a natural continuation of Classical Physics. The Model makes use a number of Hypotheses proposed by classical physicists from the 17th until the beginning of 21st century. The presented Hypotheses are not new, and we don't claim credit for them. In fact, we are developing the 
existent Hypothesis and proposing new Hypothesis in frames of WUM. The main objective of the Model is to unify and simplify existing results in Classical Physics into a single coherent picture.

WUM is a classical model. It should then be described by classical notions, which define emergent phenomena. By definition, an emergent phenomenon is a property that is a result of simple interactions that work cooperatively to create a more complex interaction. Physically, simple interactions occur at a microscopic level, and the collective result can be observed at a macroscopic level. WUM introduces classical notions, when the very first ensemble of particles was created at the cosmological time $\cong 10^{-18} \mathrm{~s}$. The World at cosmological times less than $10^{-18} \mathrm{~s}$ is best described by Quantum Mechanics [1].

In Part 2 we present principal milestones in Classical Physics and show that all the most important Fundamental Physical constants were measured and could be calculated before Quantum Mechanics. Analysis of Hypotheses proposed by classical physicists and developing them in frames of WUM are given in Part 3. In Part 4 we propose Hypotheses of Hypersphere World-Universe Model. In Part 5 Assumptions, Evidence, Principle Points and Predictions of WUM are discussed.

\section{Classical Physics}

In this Section we describe principal milestones in Classical Physics. Based on the analysis of measured physical constants we make a conclusion that the most important Fundamental constants could be calculated before Quantum Mechanics.

Kinetic Theory of Gases explains macroscopic properties of gases, such as pressure, temperature, viscosity, thermal conductivity, and volume, by considering their molecular composition and motion. In 1859, James Clerk Maxwell formulated the Maxwell distribution of molecular velocities, which gave the proportion of molecules having a certain velocity in a specific range [10]. This was the first-ever statistical law in Physics that defines macroscopic properties of gases as emergent phenomena.

Maxwell's equations were published by J. C. Maxwell in 1861 [11]. He calculated the velocity of electromagnetic waves from the value of the electrodynamic constant $c$ measured by Weber and Kohlrausch in 1857 [12] and noticed that the calculated velocity was very close to the velocity of light measured by Fizeau in 1849 [13]. This observation made him suggest that light is an electromagnetic phenomenon [14].

Rydberg constant $R_{\infty}$ is a physical constant relating to atomic spectra. The constant first arose in 1888 as an empirical fitting parameter in the Rydberg formula for the hydrogen spectral series [15]. As of 2012, $R_{\infty}$ is the most accurately measured Fundamental physical constant. The Rydberg constant can be expressed as in the following equation:

$$
R_{\infty}=\frac{\alpha^{3}}{2 a}
$$


where $\alpha$ is Sommerfeld's constant and is, in fact, the ratio of electron mass $m_{e}$ to the basic unit of mass $m_{0}: \alpha=m_{e} / m_{0}$ and $m_{0}$ equals to: $m_{0}=h / a c$, where $h$ is Planck constant, $a$ is the basic unit of length: $a=\alpha \lambda_{e}$ and $\lambda_{e}$ is the Compton wavelength of an electron: $\lambda_{e}=h / m_{e} c$.

Electron Charge-to-Mass Ratio $e / m_{e}$ is a Quantity in experimental physics. It bears significance because the electron mass $m_{e}$ cannot be measured directly. The $e / m_{e}$ ratio of an electron was successfully calculated by J. J. Thomson in 1897 [16]. We define it after Thomson: $R_{T} \equiv e / m_{e}$.

Planck Constant was suggested by Max Planck as the result of the investigations the problem of black-body radiation. He used Boltzmann's famous equation from Statistical Thermodynamics: $S=k_{B} \ln W$ that shows the relationship between entropy $S$ and the number of ways the atoms or molecules of a thermodynamic system can be arranged ( $k_{B}$ is the Boltzmann constant).

As the result of his analysis, Planck found that the average resonator entropy must be described by a function which depends on the ratios $U / v$ and $U / E$ at the same time ( $U$ is vibrational energy of vibrating resonator). Planck reconciled those two requirements through $E=h v$ in which $h$ represents a factor that converts units of frequency $v$ into units of energy $E$. Planck was able to calculate the value of $h$ from experimental data on black-body radiation: his result in $1901, h=6.55 \times 10^{-34} \mathrm{~J} \cdot \mathrm{s}$, is within $1.2 \%$ of the currently accepted value. He was also able to make the first determination of $k_{B}$ from the same data and theory: his result, $k_{B}=1.346 \times 10^{-23} \mathrm{~J} / \mathrm{K}$, is about $2.5 \%$ lower than today's figure [17].

We emphasize that Planck constant, which is generally associated with the behavior of microscopically small systems, was introduced by Max Planck based on Statistical Thermodynamics before Quantum Mechanics.

Classical Fundamental Physical Constants. Based on the experimentally measured values of the constants $R_{\infty}, R_{T}, c, h$ we calculate the most important Fundamental constants as follows:

$$
R_{T}^{2}=\left(\frac{e}{m_{e}}\right)^{2}=\frac{2 \alpha h c}{\mu_{0} m_{e}^{2} c^{2}}=\frac{2}{\alpha \mu_{0} h / c}\left(\frac{\alpha h}{m_{e} c}\right)^{2}=\frac{2}{\mu_{0} h / c} \times \frac{a^{2}}{\alpha}
$$

where $\mu_{0}$ is the magnetic constant: $\mu_{0}=4 \pi \times 10^{-7} \mathrm{H} / \mathrm{m}$. Then we can find the following equations:

$$
\begin{gathered}
R_{\infty}^{2} \times R_{T}^{2}=\frac{\alpha^{6}}{4 a^{2}} \times \frac{a^{2}}{\alpha} \times \frac{2}{\mu_{0} h / c}=\alpha^{5} \times \frac{1}{2 \mu_{0} h / c} \\
R_{\infty} \times R_{T}^{6}=\frac{\alpha^{3}}{2 a} \times \frac{a^{6}}{\alpha^{3}} \times\left(\frac{2}{\mu_{0} h / c}\right)^{3}=a^{5} \times \frac{4}{\left(\mu_{0} h / c\right)^{3}}
\end{gathered}
$$

Now we obtain:

$$
\begin{aligned}
& \alpha=\left[2\left(\mu_{0} h / c\right) R_{\infty}^{2} R_{T}^{2}\right]^{1 / 5} \\
& a=\left[\frac{\left(\mu_{0} h / c\right)^{3} R_{\infty} R_{T}^{6}}{4}\right]^{1 / 5}
\end{aligned}
$$




$$
\begin{gathered}
m_{e}=\frac{h}{c}\left[\frac{8 R_{\infty}}{\left(\mu_{0} h / c\right)^{2} R_{T}^{4}}\right]^{1 / 5} \\
e=\left(\frac{2 \alpha h / c}{\mu_{0}}\right)^{1 / 2}
\end{gathered}
$$

All these Fundamental constants, including classical electron radius $a_{o}=a / 2 \pi$, were measured and could be calculated before Quantum Mechanics.

\section{Hypotheses Revisited by WUM}

Hypersphere World-Universe Model is based on classical physics and makes use of a number of hypotheses unknown and forgotten by mainstream scientific community. Below we will describe the Hypotheses belonging to classical physicists such as Le Sage, McCullagh, Riemann, Heaviside, Bjerknes, Tesla, Dirac, and Sakharov, and develop them in frames of WUM. Please pay tribute to these great physicists!

According to WUM, two Fundamental Parameters in various rational exponents define all macro features of the World: Sommerfeld's constant $\alpha$ and dimensionless quantity $Q$. While $\alpha$ is constant, $Q$ increases with time, and is, in fact, the dimensionless Age of the World. It can be calculated from the value of the gravitational parameter $G[4]$ :

$$
Q=\frac{a^{2} c^{4}}{8 \pi h c} \times G^{-1}
$$

Three Fundamental Units define all physical dimensional parameters of the World: momentum $p_{0}=h / a$, energy density $\rho_{0}=h c / a^{4}$, and energy flux density $J_{0}=h c^{2} / a^{4}$. For all particles under consideration we use four-momentum to conduct statistical analysis of particles' ensembles, obtaining the energy density as the result. From classical point of view, we utilize three characteristics: type of particle (fermion or boson), mass, and charge.

\subsection{Aether}

Physical Aether was suggested as early as 17th century, by Isaac Newton. Following the work of Thomas Young (1804) and Augustin-Jean Fresnel (1816), it was believed that light propagates as a transverse wave within an elastic medium called Luminiferous Aether. At that time, it was realized that Aether could not be an elastic matter of an ordinary type that can only transmit longitudinal waves.

Unique properties of Aether were discussed by James McCullagh in 1846 who proposed a theory of a rotationally elastic medium, i.e. a medium in which every particle resists absolute rotation. The potential energy of deformation in such a medium depends only on the rotation of the volume elements and not on their compression or general distortion. This theory produces equations analogous to Maxwell's electromagnetic equations [18]. Aether with these properties can 
transmit transverse waves.

Luminiferous Aether was abandoned in 1905. In later years there have been classical physicists who advocated the existence of Aether:

- Nikola Tesla declared in 1937 in "Prepared Statement on the 81st birthday observance": All attempts to explain the workings of the universe without recognizing the existence of the aether and the indispensable function it plays in the phenomena are futile and destined to oblivion [19];

- Paul Dirac stated in 1951 in an article in Nature, titled “Is there an Aether?" that we are rather forced to have an aether [20].

WUM introduces the Medium of the World, which is an Aether composed of stable elementary particles: protons, electrons, photons, neutrinos, and Dark Matter particles. The existence of the Medium is a principal point of WUM. It follows from the observations of Intergalactic Plasma; Cosmic Microwave Background Radiation; Far-Infrared Background Radiation; Gamma-ray Background Radiation. According to WUM, inter-galactic voids discussed by astronomers are, in fact, examples of the Medium in its purest. The Medium is the absolute frame of reference [1].

The total energy density of the Medium $\rho_{M}$ is $2 / 3$ of the total energy density of the World $\rho_{W}$ in all cosmological times. All Macroobjects (MOs) are built from the same particles. The energy density of MOs adds up to $1 / 3$ of the total energy density throughout the World's evolution [5].

\subsection{Le Sage's Theory of Gravitation}

Wikipedia summarizes this theory as a mechanical explanation for Newton's gravitational force in terms of streams of tiny unseen particles (which Le Sage called ultra-mundane corpuscles) impacting all material objects from all directions. According to this model, any two material bodies partially shield each other from the impinging corpuscles, resulting in a net imbalance in the pressure exerted by the impact of corpuscles on the bodies, tending to drive the bodies together.

Lyman Spitzer in 1941 calculated that absorption of radiation between two dust particles leads to a net attractive force, which varies proportionally to $1 / r^{2}$ [21]. The Le Sage mechanism also has been identified as a significant factor in the behavior of dusty plasma [22].

Attempts are made to rehabilitate the theory (see, for example references [23]-[30]). In this respect, we would like to stress the importance of extended theories of gravity in the debate about gravitation, as it is clarified by C. Corda in "Interferometric detection of gravitational waves: the definitive test for General Relativity" [31]. A possibility that gravity is not an interaction, but a manifestation of a symmetry based on a Galois field is discussed by F. Lev in "Is Gravity an Interaction?" [32].

WUM introduces the Cosmic Neutrino Background (CNB), which is indeed a space-filling and isotropic flux. CNB has an energy density $\rho_{C N B}$ about $69 \%$ of the 
total energy density of the Medium $\rho_{M}$ that provides high intensity of CNB [3].

One may wonder-if there are so many neutrinos out there, how come the numerous neutrino detectors do not register them in significant quantities? Calculated Fermi energies for CNB [5] show that it consists of very low-energy neutrinos. Their interaction with matter is weak. Since the neutrino-induced cross-sections depend on the neutrinos energy linearly, such background neutrinos will not be registered by standard neutrino detectors. In fact, we might never be able to directly observe the CNB.

By analogy between Electromagnetism and Gravitoelectromagnetism, we rewrite Dirac's quantization condition for electron and monopole charges for masses $m$ and $M[5]$ :

$$
m M=0.5 M_{P}^{2}
$$

Two particles or microobjects will not exert gravity on one another when both of their masses are smaller than the Planck mass $M_{P}$. Planck mass can then be viewed as the mass of the smallest Macroobject (MO) capable of generating the gravitational field and serves as a natural borderline between classical and quantum physics [4]. It means that for the realization of Le Sage's mechanism of gravitation at least one material object must be MO. The validity of this statement follows from the work of L. Spitzer [21] and A. M. Ignatov [22].

To summarize:

- Le Sage's theory of gravitation defines Gravity as emergent phenomenon;

- Gravity is not an interaction but a manifestation of the Medium;

- The proposed mechanism of Gravitation resembles Le Sage's theory.

\subsection{Hypersphere Universe}

In 1854, Georg Riemann proposed a hypersphere as a model of a finite universe [33]. A hypersphere is the four-dimensional analog of a sphere. A regular three-dimensional sphere has a two-dimensional surface. Similarly, a 4-dimensional sphere has a 3-dimensional surface.

WUM: Before the Beginning of the World there was nothing but an Eternal Universe. About 14.2 billion years ago the World was started by a fluctuation in the Eternal Universe, and the Nucleus of the World, a 4-dimensional ball, was born. An extrapolated Nucleus radius at the Beginning was equal to $a$, that is chosen to fit the Age of the World. In WUM, a classical notion of "Size" can only be introduced when the very first ensemble of particles was created at the Nucleus radius about $a / \alpha^{2} \cong 3 \times 10^{-10} \mathrm{~m}$.

The 3D World is a hypersphere that is the surface of the 4-ball Nucleus. All points of the hypersphere are equivalent; there are no preferred centers or boundary of the World [7]. The extrapolated energy density of the World at the Beginning was four orders of magnitude smaller than the nuclear energy density [5].

The principal point of WUM is that the energy density of the World $\rho_{W}$ equals to the critical energy density $\rho_{c r}$, which can be found by considering a sphere of 
radius $R_{M}$ and enclosed mass $M$, with a small test mass $m$ on the periphery of the sphere. Mass $M$ can be calculated by multiplication of $\rho_{c r}$ by the volume of the sphere. The equation for $\rho_{c r}$ can be found from the escape speed calculation for test mass $m$ :

$$
\rho_{c r}=\frac{3 H^{2} c^{2}}{8 \pi G}=3 \rho_{0} \times Q^{-1}=\frac{3 h c}{L_{F}^{4}}
$$

where $H$ is Hubble's parameter and $L_{F}$ equals to: $L_{F}=a \times Q^{1 / 4} \quad[7]$.

\subsection{Gravitoelectromagnetism}

Gravitoelectromagnetism (GEM) is the gravitational analog of electromagnetism. The analogy and GEM equations differing from Maxwell's equations by some constants were first published by O. Heaviside in 1893 [34]. WUM follows Heaviside's approach.

\subsection{Creation of Matter}

In 1964, F. Hoyle and J. V. Narlikar offered an explanation for the appearance of new matter by postulating the existence of what they dubbed the "Creation field", or just the "C-field" [35]. In 1974, Paul Dirac discussed continuous creation of matter by additive mechanism (uniformly throughout space) and multiplicative mechanism (proportional to the amount of existing matter) [36].

WUM: 3D World is a hypersphere of 4-ball which is expanding in the Eternal Universe, so that its radius in the fourth spatial dimension is increasing with speed $c$ that is the gravitoelectrodynamic constant [5]. The lightspeed expanding hyperspherical topology was proposed in [37] [38].

The surface of the 4-ball is created in a process analogous to sublimation. It is a well-known endothermic process that occurs when surfaces are intrinsically more energetically favorable than the bulk of a material, and hence there is a driving force for surfaces to be created. Continuous creation of matter is the result of a similar process.

Matter arises from the fourth spatial dimension. The Universe is responsible for the creation of Matter. Dark Matter Particles (DMPs) carry new Matter in the World. DMPs are continuously absorbed by Dark Matter Cores of all Macroobjects (galaxy clusters, galaxies, star clusters, stars and planets) [5]. All visible Matter is re-emitted by all MOs as a result of DMPs annihilation.

It is important to emphasize that

- Creation of Matter is a direct consequence of expansion;

- Creation of Dark Matter (DM) occurs homogeneously in all points of the hypersphere World. Visible Matter is a by-product of DM annihilation. Consequently, the matter-antimatter asymmetry problem discussed in "Characterization of the 1 S-2S transition in antihydrogen" [39] does not arise.

\subsection{Multi-Component Dark Matter}

C. Boehm, P. Fayet, and J. Silk propose a way to reconcile the low and high 
energy signatures in gamma-ray spectra, even if both of them turn out to be due to Dark Matter annihilations. One would be a heavy fermion for example, like the lightest neutralino (>100 GeV), and the other one a possibly light spin-0 particle ( $100 \mathrm{MeV})$. Both of them would be neutral and also stable [40].

WUM: There are two couples of coannihilating DMPs: a heavy Dark Matter Fermion 1 (DMF1) with mass $1.3 \mathrm{TeV}$ and a light spin-0 boson-DIRAC with mass $70 \mathrm{MeV}$; a heavy fermion DMF2 with mass $9.6 \mathrm{GeV}$ and a light spin-0 boson-ELOP with mass $340 \mathrm{keV}$. Besides, we introduce a light fermion DMF3 with mass $3.7 \mathrm{keV}$. The values of DM fermion masses fall into ranges estimated in literature for neutralinos, WIMPs, and sterile neutrinos respectively [2].

WUM postulates that masses of DMPs are proportional to a basic unit of mass $m_{0}$ multiplied by different exponents of $\alpha$ :

DMF1: $m_{\mathrm{DMF} 1}=\alpha^{-2} m_{0}$

DMF2: $m_{\mathrm{DMF} 2}=\alpha^{-1} m_{0}$

DIRACs: $m_{\text {DIRAC }}=2 \alpha^{0} m_{0} / 2$

ELOPs: $m_{\mathrm{ELOP}}=2 \alpha^{1} m_{0} / 3$

DMF3: $m_{\mathrm{DMF} 3}=\alpha^{2} m_{0}$

DMF1, DMF2 and DMF3 are Majorana fermions, which partake in the annihilation interaction with strength equals to $\alpha^{-2}, \alpha^{-1}$, and $\alpha^{2}$ respectively. The signatures of DMPs annihilation with expected masses of $1.3 \mathrm{TeV} ; 9.6 \mathrm{GeV}$; $70 \mathrm{MeV} ; 340 \mathrm{keV} ; 3.7 \mathrm{keV}$ are found in spectra of the diffuse gamma-ray background and the emission of various macroobjects in the World [2]. The role of those particles in MO Cores built up from fermionic dark matter is discussed in Sections 4.2 and 4.3 .

\subsection{Macroobjects}

The existence of supermassive objects in galactic centers is now commonly accepted. Many non-traditional models explaining the supermassive dark objects observed in galaxies and galaxy clusters, formed by self-gravitating DM composed of fermions or bosons, are widely discussed in literature ([41]-[47]). The first phase of stellar evolution in the history of the World may be Dark Stars, powered by DM heating rather than fusion [48]. E. Ripamonti and T. Abel discuss the role of DM in the formation of Primordial Luminous Objects [49].

The prospect that DMPs might be observed in Centers of MOs has drawn many new researchers to the field. Indirect effects in cosmic rays and gamma-ray background from the annihilation of DM in the form of heavy stable neutral leptons in Galaxies were considered in pioneer articles [50]-[55].

WUM: All Macroobjects of the World have DM Cores surrounded by DM and baryonic shells. Annihilation of DMPs gives rise to any combination of gamma-ray lines [5].

The following facts support the existence of Cores in Macroobjects:

- A rapid rotation of the solar core has been suggested by García, et al., who also gave an approximate estimate of the solar core (below 0.2 solar radius) 
rotation rate to be between three and five times faster than that of the radiative zone [56]. More accurate results were obtained by Fossat, et al: core rotates $3.8 \pm 0.1$ faster than the radiative envelope [57];

- By analyzing the minute changes in travel times and wave shapes for earthquake doublets, Zhang, et al. concluded that the Earth's inner core is rotating faster than its surface by about 0.3 - 0.5 degrees per year [58];

- T. Guillot, et al. found that the deep interior of Jupiter rotates nearly as a rigid body, with differential rotation decreasing by at least an order of magnitude compared to the atmosphere [59].

The analysis of the Sun's heat for planets in Solar System yields the effective temperature of Earth of $255 \mathrm{~K}$ [60]. Mean surface temperature of Earth is $288 \mathrm{~K}$ [61]. The higher actual temperature of Earth is due to an energy generated internally by the planet itself. According to WUM, this energy is due to annihilation of DMPs in the Core of Earth [7].

The matter creation is occurring homogeneously in all points of the World. It follows that new stars and star clusters can be created inside of galaxies, and new galaxies and galaxy clusters can arise in the World. Structures form in parallel around different cores built from different DMPs. In WUM Dark Matter plays the main role inside of all MOs. Formation of galaxies and stars is not a process that concluded ages ago; instead, it is ongoing [3].

It is interesting to note that in 1934 Dr. Tesla stated that he is able to show that all the suns in the universe are constantly growing in mass and heat, so that the ultimate fate of each is explosion [62].

\subsection{Dirac Large Number Hypothesis}

Dirac Large Number Hypothesis is an observation made by Paul Dirac in 1937 relating ratios of size scales in the Universe to that of force scales. The ratios constitute very large, dimensionless numbers, some 40 orders of magnitude in the present cosmological epoch. According to Dirac's hypothesis, the apparent equivalence of these ratios might not to be a mere coincidence but instead could imply a cosmology where the strength of gravity, as represented by the gravitational "constant" $G$, is inversely proportional to the cosmological time $\tau$. $G \propto 1 / \tau \quad[63]$.

WUM follows the idea of time-varying $G$ and introduces a dimensionless time-varying quantity $Q$, that is the Age of the World. $G$ can be calculated from the value of the parameter $Q$ :

$$
G=\frac{a^{2} c^{4}}{8 \pi h c} \times Q^{-1}
$$

which in present epoch equals to: $Q=0.759972 \times 10^{40} \quad$ [4].

\subsection{Neutrinos}

B. Pontecorvo and Y. Smorodinsky discussed the possibility of energy density of neutrinos exceeding that of baryonic matter [64]. Neutrino oscillations imply 
that neutrinos have non-zero masses [65], [66].

WUM: According to the Model, the total energy density of neutrinos is about $69 \%$ of the critical energy density. WUM proposes the values of neutrinos mass eigenstates $m_{v_{e}}, m_{v_{\mu}}, m_{v_{\tau}}[3]$ :

$$
\begin{gathered}
m_{v_{e}}=\frac{1}{24} m_{0} \times Q^{-1 / 4} \cong 3.1 \times 10^{-4} \mathrm{eV} / \mathrm{c}^{2} \\
m_{v_{\mu}}=m_{0} \times Q^{-1 / 4} \cong 7.5 \times 10^{-3} \mathrm{eV} / \mathrm{c}^{2} \\
m_{v_{\tau}}=6 m_{0} \times Q^{-1 / 4} \cong 4.5 \times 10^{-2} \mathrm{eV} / \mathrm{c}^{2}
\end{gathered}
$$

\subsection{Emergent Gravity, Space and Time}

C. Barcelo, et al. have this to say about emergent gravity: One of the more fascinating approaches to "quantum gravity" is the suggestion, typically attributed to Sakharov [67] [68] that gravity itself may not be "fundamental physics". Indeed, it is now a relatively common opinion, that gravity (and in particular the whole notion of spacetime and spacetime geometry) might be no more "fundamental" than is fluid dynamics. The word "fundamental" is here used in a rather technical sense-fluid mechanics is not fundamental because there is a known underlying microphysics that of molecular dynamics, of which fluid mechanics is only the low-energy low-momentum limit [69].

WUM: Time and space are closely connected with Mediums' impedance and gravitomagnetic parameter. It follows that neither time nor space could be discussed in absence of the Medium. The gravitational parameter $G$ that is proportional to the Mediums' energy density can be introduced only for the Medium filled with Matter. Gravity, Space and Time are all emergent phenomena [4].

In this regard, it is worth to recall the Einstein's quote: When forced to summarize the theory of relativity in one sentence: time and space and gravitation have no separate existence from matter.

\section{Hypotheses of Hypersphere World-Universe Model}

\subsection{Dark Matter Bosons}

The quantum theory of magnetic charge started with a paper by P. Dirac in 1931 in which he showed that if any magnetic monopoles exist in the universe, then electric charge in the universe must be quantized [70]. The electric charge is, in fact, quantized, which is consistent with (but does not prove) the existence of monopoles.

WUM: We introduce DMPs DIRACs, which are dipoles of magnetic monopoles with magnetic charges $\mu=e / 2 \alpha$. They possess a substantial magnetic dipole momentum [9]. According to the Model, plasma of magnetic monopoles composes shells of star clusters' cores [6]. Such plasma can exist in a gravitational field of Macroobjects' Core.

In 1979 Haim Harari [71] and Michael A. Shupe [72] proposed a heuristic model, treating leptons and quarks as composites of spin $1 / 2$ fields with charges 
0 and $\pm e / 3$. In particle physics, preons are point particles, conceived of as subcomponents of quarks and leptons [73].

WUM: We introduce DMPs ELOPs that are dipoles of preons with electric charges $e / 3$. They have a substantial electric dipole momentum [9]. Plasma of preons composes shells of galaxies cores. It can exist in a gravitational field of Macroobjects' Core.

\subsection{Macroobject Shell Model}

According to WUM, Cores of Macroobjects of the World (galaxy clusters, galaxies, star clusters, and extrasolar systems) are Fermion Compact Stars (FCS). They have Nuclei made up of strongly annihilating dark matter fermions DMF1 or DMF2 surrounded by different shells made up of various fermions. The shells envelope one another, like a Russian doll [2]. The lighter a fermion-the greater the radius and the mass of its shell. Innermost shells are the smallest and are made up of heaviest fermions; outer shells are larger and consist of lighter particles.

The calculated parameters of the shells show that [2]:

- White Dwarf Shells around the Nuclei made of strongly annihilating DMF1 or DMF2 compose Cores of stars in extrasolar systems;

- Shells of monopoles around the Nuclei made of strongly annihilating DMF1 or DMF2 form Cores of star clusters;

- Shells of preons around the Nuclei made of strongly annihilating DMF1 or DMF2 constitute Cores of galaxies;

- Shells of DMF3 around the Nuclei made of strongly annihilating DMF1 or DMF2 make up Cores of galaxy clusters.

In our view, Macroobjects possess the following properties [6] [8]:

- Nuclei are made up of DMPs. Surrounding shells contain DM and baryonic matter;

- Nuclei and shells are growing in time proportionally to square root of cosmological time $\propto \tau^{1 / 2}$ until one of them reaches the critical point of its local instability, at which it detonates. The energy released during detonation is produced by the annihilation of DMPs. The detonation process does not destroy MO; instead, Hyper-flares occur in active regions of the shells, analogous to Solar flares;

- All other DMPs in different shells can start annihilation process as the result of the first detonation;

- Afterglow is a result of processes developing in Nuclei and shells after detonation;

- Different emission lines in spectra of bursts are connected to the Macroobjects' structure, which depends on the composition of the Nuclei and surrounding shells made up of DMPs. Consequently, the diversity of Very High Energy Bursts has a clear explanation.

In the next Section we give examples of the Macroobject Shell Model realization. 


\subsection{Multiwavelength Pulsars}

In "Gamma Ray Pulsars: Multiwavelength Observations" review D. J. Thompson presents the light curves from seven highest-confidence Gamma-Ray Pulsars (GRPs) in five energy bands: radio, optical, soft X-ray, hard X-ray/soft gamma ray, and hard gamma ray (above $100 \mathrm{MeV}$ ). Gamma rays are frequently the dominant component of the radiated power [74].

WUM: Fermi Compact Stars (FCSs) made up of strongly annihilating DMF1 and DMF2 have maximum mass and minimum size which are equal to parameters of neutron stars. It follows that GRPs might be, in fact, rotating DMF1 or DMF2 stars. The nuclei of such pulsars may also be made up of the mixture of DMF1 and DMF2 surrounded by shells composed of other DMPs. The GRP multiwavelength radiation depends on the composition of Nucleus and shells [8].

S. Ansoldi, et al. report the most energetic pulsed emission ever detected from Crab pulsar reaching up to $1.5 \mathrm{TeV}$. Such $\mathrm{TeV}$ pulsed quants require a parent population of electrons with a Lorentz factor of at least $5 \times 10^{6}$. These results strongly suggest Inverse Compton scattering of low energy photons as the emission mechanism [75].

WUM: $\mathrm{TeV}$ pulsed emission from Crab pulsar can be explained by an active area of rotating FCS composed of strongly annihilating DMF1 with mass 1.3 $\mathrm{TeV}[8]$.

Ge Chen, et al. (2015) report hard X-ray observations of the rotation-powered radio pulsar PSR B1509. The log parabolic model describes the NuSTAR data spanning $3 \mathrm{keV}$ through $500 \mathrm{MeV}$. Astronomers opinion is that the obtained results support a model in which the pulsar's lack of $\mathrm{GeV}$ emission is due to viewing geometry [76].

WUM: Multiwavelength emission from pulsar PSR B1509 can be explained by rotating DMF2 star with active area irradiating gamma quants with energy 9.6 $\mathrm{GeV}$, which interact with surrounding shells, causing them to glow in X-ray spectrum [8].

Solar flares are explosive phenomena that emit electromagnetic radiation extending from radio to gamma rays. Ackermann, M., et al. present the data of 19 solar flares detected in high-energy gamma rays in the range $60 \mathrm{MeV}$ to $6 \mathrm{GeV}$. They argue that a hadronic origin of the gamma rays is more likely than a leptonic origin [77].

WUM: Multiwavelength emission of solar flares can be explained by the annihilation of dark matter fermions DMF1 and DMF2 in the solar Core. Irradiated gamma quants with energy above $10 \mathrm{GeV}$ interact with surrounding shells, causing them to glow in a broadband spectrum.

\subsection{Electromagnetic and Gravitoelectromagnetic Parameters}

Maxwell's equations (ME) vary with the unit system used. Although the general shape remains the same, various definitions are changed, and different constants 
appear in different places. In this Section we will not rewrite well-known equations, but only provide the relationships between physical quantities used for Electromagnetism and Gravitoelectromagnetism in Table 1 and Table 2:

Table 1. Electromagnetism.

\begin{tabular}{ccc}
\hline Charge & Impedance of Electromagnetic Field & Magnetic Flux \\
\hline$q, \mathrm{C}$ & $Z_{0}=\sqrt{\frac{\mu_{0}}{\varepsilon_{0}}}=\mu_{0} c, \Omega$ & $\phi_{q}, \mathrm{~Wb}$ \\
Electric Current & Magnetic Constant & Electric Potential \\
$I_{q}, \mathrm{~A}$ & $\mu_{0}, \mathrm{H} \cdot \mathrm{m}^{-1}$ & $U_{q}, \mathrm{~V}$ \\
Magnetic Field Intensity & Electric Constant & Electric Field \\
$\boldsymbol{H}_{q}, \mathrm{~A} \cdot \mathrm{m}^{-1}$ & $\varepsilon_{0}=\left(\mu_{0} c^{2}\right)^{-1}, \phi \cdot \mathrm{m}^{-1}$ & $\boldsymbol{\boldsymbol { E } _ { q }}, \mathrm{V} \cdot \mathrm{m}^{-1}$ \\
Electric Flux Density & Electrodynamic Constant & Magnetic Flux Density \\
$\boldsymbol{D}_{q}, \mathrm{C} \cdot \mathrm{m}^{-2}$ & $c, \mathrm{~m} \cdot \mathrm{s}^{-1}$ & $\boldsymbol{B}_{q}, \mathrm{~Wb} \cdot \mathrm{m}^{-2}$ \\
\hline
\end{tabular}

Table 2. Gravitoelectromagnetism.

\begin{tabular}{ccc}
\hline Mass & Impedance of Gravitational Field & Gravitomagnetic Flux \\
\hline$m, \mathrm{~kg}$ & $Z_{g}=\sqrt{\frac{\mu_{g}}{\varepsilon_{g}}}=\mu_{g} c$ & $\phi_{m}, \mathrm{~m}^{2} \cdot \mathrm{s}^{-1}$ \\
Mass Current & Gravitomagnetic Parameter & Gravitoelectric potential \\
$I_{m}, \mathrm{~kg} \cdot \mathrm{s}^{-1}$ & $\mu_{g}=\frac{4 \pi G}{c^{2}}$ & $U_{m}, \mathrm{~m}^{2} \cdot \mathrm{s}^{-2}$ \\
Gravitomagnetic Field Intensity & Gravitoelectric Parameter & Gravitoelectric Field \\
$\boldsymbol{H}_{m}, \mathrm{~kg} \cdot \mathrm{m}^{-1} \cdot \mathrm{s}^{-1}$ & $\varepsilon_{g}=\left(\mu_{g} c^{2}\right)^{-1}$ & $\boldsymbol{E}_{m}, \mathrm{~m} \cdot \mathrm{s}^{-2}$ \\
Gravitoelectric Flux Density & Gravitoelectrodynamic Constant & Gravitomagnetic Flux Density \\
$\boldsymbol{D}_{m}, \mathrm{~kg} \cdot \mathrm{m}^{-2}$ & $c, \mathrm{~m} \cdot \mathrm{s}^{-1}$ & $\boldsymbol{B}_{m}, \mathrm{~s}^{-1}$
\end{tabular}

From the above Tables it becomes clear that the dimensions of all physical quantities depend on the choice of the charge and mass dimensions (Coulomb \& kilogram in SI units). In other unit systems the dimensions are different. For instance, in Gaussian units (CGSE): $\left[q_{e}\right]=\mathrm{cm}^{3 / 2} \cdot \mathrm{g}^{1 / 2} \cdot \mathrm{s}^{-1}$ and in CGSM: $\left[q_{m}\right]=\mathrm{cm}^{1 / 2} \cdot \mathrm{g}^{1 / 2}$.

We seem to possess a substantial degree of freedom when it comes to choosing the dimension of charge and mass. For an arbitrary dimension-transposing parameter $P$ we can

- Multiply the charge and mass and all physical quantities on the left side of Table 1 and Table 2 by an arbitrary parameter $P$,

- Divide impedances by $P^{2}$;

- Divide magnetic fluxes and all physical quantities on the right side of Table 1 and Table 2 by $P$.

Following such a transformation, all physically measurable parameters such as energy density and energy flux density remain the same and have the same mechanical dimensions. 
There are two physical sources in ME: total electric charge density $\rho_{q}$ and total electric current density $J_{q}$. According to ME, there are two measurable physical characteristics: energy density $\rho_{E}$ and energy flux density $J_{E}$. It is interesting to proceed with $\mathrm{ME}$ when physical sources are energy density $\rho_{E}$ and energy flux density $\boldsymbol{J}_{E}$, which coincide with the same measurable physical characteristics. It means that electromagnetic and gravitoelectromagnetic charges should have the dimension of "Energy" [9]. Below we make transformations for magnetic parameter of the Medium resulting in the dimension of electromagnetic and gravito electromagnetic charges "Area" that is equivalent to "Energy" with a constant$\sigma_{0}$, that is a basic unit of surface energy density: $\sigma_{0}=\frac{h c}{a^{3}}$.

In frames of WUM, the gravitational parameter $G$ can be calculated based on the value of the energy density of the Medium $\rho_{M}[1]$ :

$$
G=\frac{\rho_{M}}{4 \pi} \times P_{g}^{2}
$$

where a dimension-transposing parameter $P_{g}$ equals to:

$$
P_{g}=\frac{a^{3}}{2 h / c}
$$

Using the flexibility of gravitoelectromagnetic charge dimension we replace mass $m$ with

$$
m \times P_{g}=a^{3} / 2 L_{C m}
$$

where $L_{c m}$ is Compton length of mass $m$. The gravitoelectromagnetic charge has a dimension of "Area", which is equivalent to "Energy" with the constant $2 \sigma_{0}$. Then Newton's law of universal gravitation can be rewritten in the following way:

$$
F=G \frac{m \times M}{r^{2}}=\frac{\rho_{M}}{4 \pi} \frac{\frac{a^{3}}{2 L_{C m}} \times \frac{a^{3}}{2 L_{C M}}}{r^{2}}
$$

where we introduce the measurable parameter of the Medium $\rho_{M}$ instead of the phenomenological coefficient $G$; and gravitoelectromagnetic charges $\frac{a^{3}}{2 L_{C m}}$ and $\frac{a^{3}}{2 L_{C M}}$ instead of masses $m$ and $M$. We took constant $2 \sigma_{0}$ to fit the total energy of masses $m$ and $M$ :

$$
\frac{a^{3}}{2 L_{C m}} \times 2 \sigma_{0}=\frac{a^{3} m c}{2 h} \times \frac{2 h c}{a^{3}}=m c^{2}
$$

As the result of this transformation, the gravitomagnetic parameter $\mu_{g}=\frac{4 \pi G}{c^{2}}$ transforms into $\mu_{g g}$ :

$$
\mu_{g g}=\rho_{M} / c^{2}
$$


that is precisely equals to the energy density of the Medium over $c^{2}$ [4].

For free electric charges $e$ in the Medium of the World we use a dimensiontransposing parameter $P_{e}=\frac{e_{g}}{e}$ where $e_{g}$ equals to: $e_{g}=4 \pi\left(\frac{L_{F}}{2 \pi}\right)^{2} \cdot e_{g}$ has a dimension of "Area", which is equivalent to "Energy" with the constant $2 \sigma_{0}$. Then magnetic parameter $\mu_{0}=\frac{2 \alpha h}{c e^{2}}$ transforms into $\mu_{0 g}$ :

$$
\mu_{o g}=\frac{2 \alpha h}{c e_{g}^{2}}=\frac{2 \pi^{2} \alpha}{3} \frac{\rho_{c r}}{c^{2}}=\frac{\rho_{p}}{c^{2}}
$$

$\mu_{0 g}$ precisely equals to the value of proton energy density in the Medium $\rho_{p}$ over $c^{2}[1]$.

It follows that we can treat the electromagnetic field with constant magnetic parameter $\mu_{0}$ in the time-varying gravitational Medium with the magnetic parameter $\mu_{0 g} \propto \tau^{-1}$ and a time-varying electric charge $e_{g} \propto \tau^{1 / 2}$. In this case, free electric charges in the Medium can be treated as the pulsating spheres with the radius $\frac{L_{F}}{2 \pi} \cong 2.63 \times 10^{-5} \mathrm{~m}$.

This approach aligns WUM with Bjerknes mechanism for the attraction and the repulsion between two pulsating spheres. Lord Kelvin and Carl Anton Bjerknes investigated this mechanism between 1870 and 1903. Bjerknes showed that when two spheres immersed in a fluid were pulsating, they exerted a mutual attraction, which obeyed Newton's inverse square law if the pulsations are in phase. The spheres repelled when the phases differed by a half wave [78].

\subsection{Modified Maxwell's Equations}

To apply ME, it is necessary to specify the constitutive relations:

$$
\begin{aligned}
\boldsymbol{D} & =\varepsilon_{0} \boldsymbol{E} \\
\boldsymbol{H} & =\frac{1}{\mu_{0}} \boldsymbol{B} \\
\boldsymbol{E} & =\rho_{r} \boldsymbol{J}
\end{aligned}
$$

where $\varepsilon_{0}$ is the electric constant and $\rho_{r}$ is the electric current resistivity that we propose to take the value of $\rho_{r}=\mu_{0} c a=Z_{0} a$. We emphasize that all quantities in ME can be calculated based on physical sources $\rho$ and $\boldsymbol{J}$ [9].

There are two auxiliary field quantities:

$$
\begin{gathered}
\boldsymbol{D}=\varepsilon_{0} \boldsymbol{E}+\boldsymbol{P} \\
\boldsymbol{H}=\frac{1}{\mu_{0}} \boldsymbol{B}-\boldsymbol{M}
\end{gathered}
$$

The quantities $\boldsymbol{P}$ and $\boldsymbol{M}$ represent the macroscopically averaged electric dipole and magnetic dipole moment densities of the material medium in the presence of applied fields. Analysis of ME in which all quantities are arbitrary functions of space and time has been done in literature ([79] [80]). 
K. Brown discusses two classical models for the source of "magnetic dipole" fields: one based on the juxtaposition of two oppositely charged magnetic monopoles, and one based on a loop of electric current. These two models might be called Coulombic and Amperean dipoles respectively.

He emphasizes the difference between $\boldsymbol{B}$ and $\boldsymbol{H}$ fields. Outside any magnetic material, $\boldsymbol{B}$ and $\boldsymbol{H}$ are strictly proportional to each other, but inside magnetic material they are quite different. The potential energy density of a magnetic field is really $(\boldsymbol{B} \cdot \boldsymbol{H}) / 2$, and reduces to $\boldsymbol{B}^{2} / 2 \mu_{0}$ only outside of any magnetic material [81].

M. Mansuripur compared two versions of the Poynting vector $\boldsymbol{S}=(\boldsymbol{E} \times \boldsymbol{B}) / \mu_{0}$ and $\boldsymbol{S}=\boldsymbol{E} \times \boldsymbol{H}$. He concludes: the identification of magnetic dipoles with Amperian current loops, while certainly acceptable within the confines of Maxwell s macroscopic equations, is inadequate and leads to complications when considering energy, force, torque, momentum, and angular momentum in electromagnetic systems that involve the interaction of fields and matter [79].

WUM: The Medium of the World consists of the following elementary particles [7]:

- Protons and electrons with mass $m_{p}$ and $m_{e}$ and electric charge $e$,

- Mass-varying neutrinos and photons;

- DMPs including fermions (DMF1, DMF2, DMF3) and bosons (DIRACs and ELOPs);

- ELOPs with mass $2 m_{e} / 3$ that are electric dipoles of preons with electric charges e/3. They represent the macroscopically averaged electric dipole moment density $\boldsymbol{P}$ of the Medium in the presence of applied fields with energy density about the proton energy density [7];

- DIRACs with mass $m_{0}$, which are magnetic dipoles of Dirac's monopoles with magnetic charges $\mu=e / 2 \alpha$. DIRACs are the Coulombic magnetic dipoles. Their energy density in the Medium is about the proton energy density [7]. They represent the macroscopically averaged magnetic dipole moment density $M$ of the Medium in the presence of applied fields.

It is well-known that the dimension of the magnetic field intensity $[\boldsymbol{H}]=\mathrm{A} \cdot \mathrm{m}^{-1}$. We can rewrite it in the following way:

$$
[\boldsymbol{H}]=\frac{\mathrm{C} \cdot \mathrm{m}}{\mathrm{m}^{2} \cdot \mathrm{s}}=\frac{\left[\boldsymbol{d}_{m}\right]}{\mathrm{m}^{2} \cdot \mathrm{s}}=\left[\boldsymbol{J}_{m}\right]
$$

where $\boldsymbol{d}_{m}$ is a magnetic dipole momentum. It looks like magnetic field intensity $H$ is, in fact, proportional to the current density $\boldsymbol{J}_{m}$ of magnetic dipoles $d_{m}$ :

$$
\boldsymbol{H}=\rho_{m} \boldsymbol{J}_{m}
$$

where $\rho_{m}$ is a magnetic dipole current resistivity. In our opinion, the magnetic field intensity $H$ is not an "auxiliary" field quantity. On the contrary, it is a real magnetic field quantity. That is why $\boldsymbol{S}=\boldsymbol{E} \times \boldsymbol{H}$ is a true Poynting vector and 
$(\boldsymbol{B} \cdot \boldsymbol{H}) / 2$ is a true potential energy density of a magnetic field.

In summary: magnetic monopoles are not the subject of Maxwell's equations; instead, magnetic dipoles DIRACs are. To describe the propagation of electromagnetic signals through the Medium of the World we should modify Maxwell's equations [9]:

- consider the macroscopically averaged electric $\boldsymbol{P}$ and magnetic $\boldsymbol{M}$ dipole moment density of the Medium in the presence of applied fields;

- consider ELOPs and DIRACs current densities induced by the electromagnetic field.

Most articles on electromagnetic theory follow the classical approach of steady state solutions of Maxwell's equations. H. Harmuth and K. Lukin in "Interstellar Propagation of Electromagnetic Signals" point out the deficiencies in Maxwell's theory and present an exciting new way of obtaining transient or signals solutions. A new approach based on microscopic description of the medium and analytical solution of Maxwell equations in time domain has been used to solve the problem [82].

H. Harmuth and K. Lukin analyzed the propagation of electromagnetic signals through a non-conducting medium with very low density of neutral gas considering both electric and magnetic dipole currents [82]. Authors modify Maxwell's equations for "empty space" using both electric and magnetic dipole current densities rather than electric and magnetic flux densities. This implies description of the medium in the frame of microscopic approach using representation of a hydrogen atom as a combination of electric and magnetic dipoles [82]. Those dipoles produce electric and magnetic dipole currents under the electromagnetic field action that is to be calculated in a self-consistent way.

$\mathrm{H}$. Harmuth and K. Lukin created a self-consistent system containing both Maxwell equations and equations for the dipole current densities evolution under the electromagnetic field action in the following form [83]:

$$
\begin{aligned}
&-r o t \boldsymbol{E}=\mu \frac{\partial \boldsymbol{H}}{\partial t}+\boldsymbol{g}_{m} \\
& r o t \boldsymbol{H}=\varepsilon \frac{\partial \boldsymbol{E}}{\partial t}+\boldsymbol{g}_{e} \\
& \varepsilon d i v \boldsymbol{E}=\mu \operatorname{div} \boldsymbol{H}=0 \\
& \boldsymbol{g}_{e}+\tau_{m p} \frac{\partial \boldsymbol{g}_{e}}{\partial t}+\frac{\tau_{m p}}{\tau^{2}} \int \boldsymbol{g}_{e} \mathrm{~d} t=\sigma_{p} \boldsymbol{E} \\
& \boldsymbol{g}_{m}+\tau_{m p} \frac{\partial \boldsymbol{g}_{m}}{\partial t}+\frac{\tau_{m p}}{\tau^{2}} \int \boldsymbol{g}_{e} \mathrm{~d} t=2 s_{p} \boldsymbol{H} \\
& \sigma_{p}=\frac{N_{0} e^{2} \tau_{m p}}{m} \\
& s_{p}=\frac{N_{0} q_{m}^{2} \tau_{m p}}{m} \\
& q_{m}=\frac{\mu m_{m 0}}{2 r}
\end{aligned}
$$


where $\sigma_{p}$ and $s_{p}$ are electric and magnetic dipole current conductances; $\boldsymbol{g}_{e}$ and $\boldsymbol{g}_{m}$ are electric and magnetic dipole current densities induced by the electromagnetic field; $\varepsilon$ and $\mu$ are electric and magnetic constants; $e$ and $m$ are charge and mass of an electron; $m_{m 0}$ and $q_{m}$ are magnetic dipole moment and fictitious magnet charge; $\tau_{m p}$ and $\tau$ are the relaxation time and period of eigen-frequency of the dipole-oscillator used as the model for atomic hydrogen.

Authors concluded that the time delay between the signal precursor and its main lobe evaluated may be used for evaluation of either distance to a pulsar for the known medium parameters or those parameters for a given distance to the pulsar [83].

R. Beck and R. Wielebinski discuss the omnipresence of Cosmic Magnetism: Most of the visible matter in the Universe is ionized, so that cosmic magnetic fields are quite easy to generate and due to the lack of magnetic monopoles hard to destroy. Magnetic fields have been measured in or around practically all celestial objects. The Earth, the Sun, solar planets, stars, pulsars, the Milky Way, nearby galaxies, more distant (radio) galaxies, quasars and even intergalactic space in clusters of galaxies have significant magnetic fields, and even larger volumes of the Universe may be permeated by "dark" magnetic fields [84].

WUM explains the similarity of field patterns and flow patterns of the diffuse ionized gas [85] by the flow of DIRACs along with diffuse ionized gas. The large-scale structure of the Milky Way's magnetic field [85], a dark magnetic field [86] and other magnetic phenomena which are only partly related to objects visible in other spectral ranges [84] can be explained by flows of dark matter particles DIRACs. We believe that the developed approach to magnetic field [9] can answer questions on the origin and evolution of magnetic fields such as their first occurrence in young galaxies, or the existence of large-scale intergalactic fields [84].

In conclusion:

- We should build an all-encompassing theory of Intergalactic propagation of electromagnetic signals considering the content of the Medium proposed in WUM;

- Hypersphere WUM can serve as a basis for Cosmic Magnetism.

\subsection{Inter-Connectivity of Primary Cosmological Parameters}

The constancy of the universe fundamental constants, including Newtonian constant of gravitation, Fermi coupling constant, Planck mass, is now commonly accepted, although has never been firmly established as a fact. All conclusions on the (almost) constancy of the Newtonian parameter of gravitation are model-dependent [4]. A commonly held opinion states that gravity has no established relation to other fundamental forces, so it does not appear possible to calculate it indirectly from other constants that can be measured more accurately, as is done in some other areas of physics. WUM holds that there indeed exist relations between all primary cosmological parameters that depend on dimen- 
sionless time-varying quantity $Q[5]$.

The model develops a mathematical framework that allows for direct calculation of the following parameters through $Q$ [7]:

- Newtonian parameter of gravitation $G$,

- Hubble's parameter $H$;

- Age of the World $A_{r}$;

- The Worlds' radius of curvature in the fourth spatial dimension $R$;

- Critical energy density $\rho_{c r}$

- Temperature of the Microwave Background Radiation $T_{M B R}$;

- Temperature of the Far-Infrared Background Radiation peak $T_{F I R B}$;

- Electronic neutrino mass $m_{v_{e}}$;

- Muonic neutrino mass $m_{v_{\mu}}$;

- Tauonic neutrino mass $m_{v_{\tau}}$;

- Fermi coupling parameter $G_{\vec{P}}$

- Photons minimum energy $E_{p h r}$.

The precision of their measured values increases by orders of magnitude. In frames of WUM, we calculate the values of these parameters, which are in good agreement with the latest results of their measurements.

For example, calculating the value of Hubble's parameter $H_{0}$ based on the average value of the gravitational parameter $G$ we find $H_{0}=68.7457 \mathrm{~km} / \mathrm{s} \cdot \mathrm{Mpc}$, which is in good agreement with $H_{0}=69.32 \pm 0.8 \mathrm{~km} / \mathrm{s} \cdot \mathrm{Mpc}$ obtained using WMAP data [87].

The black-body spectrum of the cosmic Microwave Background Radiation (MBR) is due to thermodynamic equilibrium of photons with low density intergalactic plasma [1]. WUM calculates the value of $T_{M B R}$ to be $T_{M B R}=2.72518 \mathrm{~K}$, which is in excellent agreement with experimentally measured value of 2.72548 $\pm 0.00057 \mathrm{~K}[88]$.

Based on the thermo-equilibrium of drops of Bose-Einstein-condensed dineutrinos [3] we calculate their stationary temperature that corresponds to the Far-Infrared Background temperature peak $T_{F I R B}$ and obtain $T_{F I R B}=28.955 \mathrm{~K}$, which is in an excellent agreement with experimentally measured value of $29 \mathrm{~K}$ ([89]-[100]).

L. Zyga has found that the measured $G$ values from 1980 to 2015 oscillate over time (about $\pm 0.001 \times 10^{-11} \mathrm{~m}^{3} \cdot \mathrm{kg}^{-1} \cdot \mathrm{s}^{-2}$ ) like a sine wave with a period of 5.9 years [101]. In frames of WUM, these results can be explained by variations of the flux of neutrinos emanating from Sun.

Today, Fermi coupling parameter is known with the highest precision. Based on its average value:

$$
G_{F}=1.1663787 \times 10^{-5} \mathrm{GeV}^{-2}
$$

we can calculate and significantly increase the precision of the values of all $Q$-dependent parameters. The calculated value of the parameter $Q_{F}$ based on $G_{F}$ is [4]: 


$$
Q_{F}=0.75992106 \times 10^{40}
$$

that is much more precise than the value of parameter $Q_{G}$ calculated based on $G$ :

$$
Q_{G}=0.759972 \times 10^{40}
$$

As an example of the increased precision:

- the measured average value of $G$ from CODATA is:

$$
G=6.67408 \times 10^{-11} \mathrm{~m}^{3} \cdot \mathrm{kg}^{-1} \cdot \mathrm{s}^{-2}
$$

- the calculated value of $G$ based on $Q_{F}$ is:

$$
G=6.6745358 \times 10^{-11} \mathrm{~m}^{3} \cdot \mathrm{kg}^{-1} \cdot \mathrm{s}^{-2}
$$

The CODATA value of $G$ is slightly smaller $(<0.007 \%)$ than the calculated value.

WUM makes reasonable assumptions in the main areas of Cosmology. The remarkable agreement of the calculated values of the primary cosmological parameters with the observational data gives us considerable confidence in the Model. We propose to introduce $Q$ as a new Fundamental Parameter tracked by CODATA and use its value in calculation of all $Q$-dependent parameters.

\section{Assumptions, Evidence, Principle Points and Predictions}

\subsection{Assumptions}

WUM is based on the following primary assumptions [1]:

- The universality of physical laws;

- The cosmological principle which states that on a large scale the World is homogeneous and isotropic;

- The World is a finite three-dimensional Hypersphere. All points of the Hypersphere are equivalent; there are no preferred centers or boundary of the World;

- The World is expanding inside the Universe along the fourth spatial dimension with speed equal to the gravitoelectrodynamic constant $c$,

- Supremacy of Matter and continuous creation of Matter;

- Variable Gravitational parameter;

- Maxwell's equations for Electromagnetism and Gravitoelectromagnetism;

- Elementary particles have the following characteristics: type of particle (fermion or boson), four-momentum, mass and charge;

- The Medium of the World, consisting of protons, electrons, photons, neutrinos, and dark matter particles is an active agent in all physical phenomena in the World.

\subsection{Evidence of the Hypersphere World}

The physical laws we observe appear to be independent of the Worlds' curvature in the fourth spatial dimension due to small enough observers in comparison with the radius of the curvature. Direct observation of the Worlds' curvature would then appear to be a hopeless goal. One way to prove the existence of the Worlds' curvature is direct measurement of truly large-scale parameters of the 
World: Gravitational, Hubble's, Temperature of the Microwave Background Radiation. Conducted at various points of time, these measurements would give us varying results, providing insight into the curved nature of the World. Unfortunately, the accuracy of the measurements is quite poor. Measurement errors far outweigh any possible "curvature effects", rendering this technique useless in practice. To be conclusive, the measurements would have to be conducted billions of years apart.

Let's consider an effect that has indeed been observed for billions of years, albeit indirectly [5]. 4.6 billion years ago the Suns' output has been only 70 percent as intense during that epoch as it is during the modern epoch [102]. One of the consequences of WUM holds that all stars were fainter in the past. As their cores absorb new DM, size of macroobjects cores $R_{M O}$ and their luminosity $L_{M O}$ are increasing in time $R_{M O} \propto Q^{1 / 2} \propto \tau^{1 / 2}$ and $L_{M O} \propto Q \propto \tau$ respectively. Taking the Age of the World $\cong 14.2 \mathrm{Byr}$ and the age of solar system $\cong 4.6 \mathrm{Byr}$, it is easy to find that the young Suns' output was $67 \%$ of what it is today [2].

Another effect that has been observed directly is photons' time delay relative to the light travel time $t_{L T T}=\tau_{\text {obsv }}-\tau_{\text {emit }}$ from the source of Fast Radio Burst (FRB) billions of years away from Earth. $\tau_{\text {emit }}$ and $\tau_{\text {obsv }}$ are cosmological times (Ages of the World at the moments of emitting and observing photons), both measured from the Beginning of the World [6]. FRBs are bright, unresolved, broadband, millisecond flashes found in parts of the sky outside Milky Way. Astronomers believe that the pulses are emitted simultaneously over a wide range of frequencies. However, as observed on Earth, the components of each pulse emitted at higher radio frequencies arrive before those emitted at lower frequencies. This delay is described by a value referred to as a Dispersion Measure which depends on the number density of electrons integrated along the path traveled by the photon from the source of FRB to Earth [103] [104].

We propose to calculate a Dispersion Measure based on the calculated electron concentration $n_{e}$ in the Medium of the World that decreasing in time $n_{e} \propto Q^{-1} \propto \tau^{-1} \quad$ [1]. The calculated value of photons' time delay for FRB 150418 [6] is in good agreement with experimentally measured value [105]. We emphasize that the described astrophysical phenomenon, Fast Radio Burst, manifests the existence of the Intergalactic plasma.

The proposed approach to the fourth spatial dimension agrees with Mach's principle: "Local physical laws are determined by the large-scale structure of the universe". Applied to WUM, it follows that all parameters of the World depending on $Q$ are a manifestation of the Worlds' curvature in the fourth spatial dimension [1].

\subsection{Principle Points}

WUM is based on the following Principle Points [5]:

- The World was started by a fluctuation in the Eternal Universe, and the Nucleus of the World, which is a four dimensional 4-ball, was born. The Begin- 
ning of the World is a Quantum effect.

- The 3D World is the Hypersphere that is the surface of the 4-ball Nucleus. Hence the World is curved in the fourth spatial dimension.

- The 4-ball is expanding in the Eternal Universe, and its surface, the hypersphere, is likewise expanding so that the radius of the 4-ball is increasing with speed $c$ that is the gravitoelectrodynamic constant.

- The surface of the hypersphere is created in a process analogous to sublimation, which is an endothermic process. Continuous creation of matter is the result of a similar process. The creation of matter is happening homogeneously in all points of the hypersphere World and is a direct consequence of expansion. Visible Matter is a by-product of DM annihilation.

- The World consists of the Medium and Macroobjects. The Medium consists of stable elementary particles with lifetimes longer than the age of the World: protons, electrons, photons, neutrinos, and dark matter particles. The energy density of the Medium is $2 / 3$ of the total energy density in all cosmological times.

- Galaxy clusters, Galaxies, Star clusters, Extrasolar systems, Planets, etc. are made of these particles. The energy density of Macroobjects is $1 / 3$ of the total energy density throughout the World's evolution.

- Time, Space and Gravitation are emergent phenomena and have no separate existence from Matter. In WUM, they are closely connected with the impedance, the gravitomagnetic parameter, and the energy density of the Medium respectively.

- Maxwell's Equations for Electromagnetism and Gravitoelectromagnetism play a principal role in the description of the World.

- Two Fundamental Parameters in various rational exponents define all macro features of the World: Sommerfeld's constant $\alpha$ and dimensionless Quantity $Q$. While $\alpha$ is constant, $Q$ increases in time, and is, in fact, a measure of the Worlds' curvature in the fourth spatial dimension and the Age of the World.

- WUM holds that there exist relations between all $Q$-dependent parameters: Newtonian parameter of gravitation and Hubble's parameter; Critical energy density and Fermi coupling parameter; Temperatures of the Microwave Background Radiation and Far-Infrared Background Radiation peak. The calculated values of these parameters are in good agreement with the latest results of their measurements. Model proposes to introduce a new fundamental quantity $Q$ in the CODATA internationally recommended values for calculating all $Q$-dependent parameters of the World.

- The black-body spectrum of the cosmic Microwave Background Radiation is due to thermodynamic equilibrium of photons with low density Intergalactic Plasma.

- The Far-Infrared Background Radiation is due to the emission of BEC drops created as the result of the Bose-Einstein Condensation (BEC) of dineutrinos. The BEC drops absorb energy directly from the Medium of the World pro- 
vided by dineutrinos and re-emit this energy in FIRB at the stationary temperature $T_{F I R B}$.

- The total energy density of neutrinos is about $69 \%$ of the critical energy density.

- Dark Matter (DM) consists of 5 different particles: DMF1, DMF2, DMF3, DIRACs, and ELOPs, and has the relative energy density of about $24 \%$ [2].

- All Macroobjects of the World (galaxy clusters, galaxies, star clusters, extrasolar systems, and planets) possess the following properties: their Cores are made up of DMPs; they contain other particles, including DM and baryonic matter, in shells surrounding the Cores. Annihilation of DMPs can give rise to any combination of gamma-ray lines.

- The total cosmic-ray radiation consists of Gamma-ray Background Radiation plus X-ray radiation from the different highly ionized chemical elements in the hot areas of the World [2].

- Nucleosynthesis of all elements occurs inside stars during their evolution. Stellar nucleosynthesis theory should be enhanced to account for annihilation of heavy DMPs (DMF1 and DMF2) inside of the Stars' Cores [5].

- Macroobjects form from top (galaxy clusters) down to extrasolar systems in parallel around different Cores made of different DMPs. Formation of galaxies and stars is not a process that concluded ages ago; instead, it is ongoing.

- Assuming an Eternal Universe, the numbers of cosmological structures on all levels will increase: new galaxy clusters will form; existing clusters will obtain new galaxies; new stars will be born inside existing galaxies; sizes of individual stars will increase, etc. The temperature of the Medium of the World will asymptotically approach absolute zero [5].

\subsection{Predictions}

WUM makes the following predictions, which we hope will be supported by experimental data [5]:

- All Macroobjects of the World (galaxy clusters, galaxies, star clusters, extrasolar systems, and planets) possess Cores that are made up of DMPs. All round objects in hydrostatic equilibrium, down to Mimas in Solar system, should be considered Planets;

- WUM predicts existence of DMPs with $1.3 \mathrm{TeV}, 9.6 \mathrm{GeV}, 70 \mathrm{MeV}, 340 \mathrm{keV}$, and $3.7 \mathrm{keV}$ masses;

- Model makes predictions pertaining to neutrinos mass eigenstates and photons minimum energy in a present cosmological epoch:

$m_{v_{e}} \cong 3.1 \times 10^{-4} \mathrm{eV} / \mathrm{c}^{2} ; m_{v_{\mu}} \cong 7.5 \times 10^{-3} \mathrm{eV} / \mathrm{c}^{2} ; \quad m_{v_{\tau}} \cong 4.5 \times 10^{-2} \mathrm{eV} / \mathrm{c}^{2} \quad$ and $E_{\text {phi }} \cong 1.9 \times 10^{-14} \mathrm{eV}$ respectively [5];

- WUM predicts the concentration of Intergalactic plasma in the present cosmological epoch: $n_{p}=n_{e}=0.2548 \mathrm{~m}^{-3} \quad[1]$;

- Model proposes new types of particle interactions (Super Weak and Extremely Weak) with coupling strength in the present cosmological epoch: 
$\sim 10^{-10}$ and $\sim 10^{-20}$ times weaker than that of weak interaction [3].

The Hypersphere World-Universe Model successfully describes primary parameters and their relationships, ranging in scale from cosmological structures to elementary particles. WUM allows for precise calculation of values that were only measured experimentally earlier and makes verifiable predictions. WUM does not attempt to explain all available cosmological data, as that is an impossible feat for any one manuscript. Nor does WUM pretend to have built an all-encompassing theory that can be accepted as is. The Model needs significant further elaboration, but in its present shape, it can already serve as a basis for a new Classical Physics proposed by Paul Dirac in 1937. The Model should be developed into a well-elaborated theory by all physical community.

\section{Acknowledgements}

I am a Doctor of Sciences in Physics. I belong to the school of physicists established by Alexander Prokhorov-Nobel Prize Laureate in Physics. I'm an author of more than 150 published papers. I'm very grateful to Prof. A. M. Prokhorov and Prof. A. A. Manenkov whose influence on my scientific life is decisive.

Many thanks to my long-term friend Felix Lev for stimulating discussions of history and philosophy of Physics and important comments on the Model.

Special thanks to my son Ilya Netchitailo, who questioned every aspect of the Model, gave valuable suggestions and helped shape it to its present form.

\section{References}

[1] Netchitailo, V.S. (2015) 5D World-Universe Model Space-Time-Energy. Journal of High Energy Physics, Gravitation and Cosmology, 1, 25-34. https://doi.org/10.4236/jhepgc.2015.11003

[2] Netchitailo, V.S. (2015) 5D World-Universe Model. Multicomponent Dark Matter. Journal of High Energy Physics, Gravitation and Cosmology, 1, 55-71. https://doi.org/10.4236/jhepgc.2015.12006

[3] Netchitailo, V.S. (2016) 5D World-Universe Model. Neutrinos. The World. Journal of High Energy Physics, Gravitation and Cosmology, 2, 1-18. https://doi.org/10.4236/jhepgc.2016.21001

[4] Netchitailo, V.S. (2016) 5D World-Universe Model. Gravitation. Journal of High Energy Physics, Gravitation and Cosmology, 2, 328-343. https://doi.org/10.4236/jhepgc.2016.23031

[5] Netchitailo, V.S. (2016) Overview of Hypersphere World-Universe Model. Journal of High Energy Physics, Gravitation and Cosmology, 2, 593-632. https://doi.org/10.4236/jhepgc.2016.24052

[6] Netchitailo, V.S. (2017) Burst Astrophysics. Journal of High Energy Physics, Gravitation and Cosmology, 3, 157-166. https://doi.org/10.4236/jhepgc.2017.32016

[7] Netchitailo, V.S. (2017) Mathematical Overview of Hypersphere World-Universe Model. Journal of High Energy Physics, Gravitation and Cosmology, 3, 415-437. https://doi.org/10.4236/jhepgc.2017.33033

[8] Netchitailo, V.S. (2017) Astrophysics: Macroobject Shell Model. Journal of High Energy Physics, Gravitation and Cosmology, 3, 776-790. https://doi.org/10.4236/jhepgc.2017.34057 
[9] Netchitailo, V.S. (2018) Analysis of Maxwell's Equations. Cosmic Magnetism. Journal of High Energy Physics, Gravitation and Cosmology, 4, 1-7. https://doi.org/10.4236/jhepgc.2018.41001

[10] Maxwell, J.C. (1860) Illustrations of the Dynamical Theory of Gases. The London, Edinburgh, and Dublin Philosophical Magazine and Journal of Science, 20, 21-37. https://doi.org/10.1080/14786446008642902

[11] Maxwell, J.C. (1861) On Physical Lines of Force. Philosophical Magazine, 90, 11-23. https://doi.org/10.1080/14786431003659180

[12] Kohlrausch, R. and Weber, W. (1857) Elektrodynamische Maaßbestimmungen: Insbesondere Zurückfuhrung der Stromintensitats-Messungen auf mechanisches Maass. On the Amount of Electricity which Flows through the Cross-Section of the Circuit in Galvanic Currents (Translated by Susan P. Johnson and edited by Laurence Hecht).

http://ppp.unipv.it/Collana/Pages/Libri/Saggi/Volta\%20and\%20the\%20History\%20 of\%20Electricity/V\%26H\%20Sect3/V\%26H\%20287-297.pdf

[13] Fizeau, H. (1849) Comptes Rendus: Hebdomadaires de scéances de l'Academie de Sciences (Paris, Vol. 29 [July-December 1849], pp. 90-92).

[14] Maxwell, J.C. (1865) A Dynamical Theory of the Electromagnetic Field. Philosophical Transactions of the Royal Society of London, 155, 459-512. https://doi.org/10.1098/rstl.1865.0008

[15] Heüman, G.D. (1888) The Rydberg Formula as Presented to Matematiskt-Fysiska förening. https://commons.wikimedia.org/wiki/File:Rydbergformula.jpg

[16] Thomson, J.J. (1897) Cathode Rays. Philosophical Magazine, 44, 293. http://web.lemoyne.edu/ giunta/thomson1897.html

[17] Plank, M. (1901) On the Law of Distribution of Energy in the Normal Spectrum. Annalen der Physik, 4, 553.

https://web.archive.org/web/20080418002757/http://dbhs.wvusd.k12.ca.us/webdocs /Chem-History/Planck-1901/Planck-1901.html

[18] MacCullagh, J. (1846) An Essay towards a Dynamical Theory of Crystalline Reflexion and Refraction. Transactions of the Royal Irish Academy, 21, 17-50.

[19] Tesla, N. (1937) Prepared Statement on the 81st Birthday Observance. http://www.institutotesla.org/tech/TeslaGravity.html

[20] Dirac, P.M. (1951) Is There an Aether? Nature, 168, 906-907.

[21] Spitzer, L. (1941) The Dynamics of the Interstellar Medium; II. Radiation Pressure. The Astrophysical Journal, 94, 232. https://doi.org/10.1086/144328

[22] Ignatov, A.M. (1996) Lesage Gravity in Dusty Plasma. Plasma Physics Reports, 22, 58.

[23] Radzievskii, V.V. and Kagalnikova, I.I. (1960) The Nature of Gravitation. Bulletin of the All-Union Astronomical-Geodetic Society, 26, 3-14.

[24] Shneiderov, A.J. (1961) On the Internal Temperature of the Earth. Bollettino di Geofisica Teorica ed Applicata, 3, 137.

[25] Buonomano, V. and Engel, E. (1976) Some Speculations on a Causal Unification of Relativity, Gravitation, and Quantum Mechanics. International Journal of Theoretical Physics, 5, 231-246. https://doi.org/10.1007/BF01807095

[26] Adamut, I.A. (1982) The Screen Effect of the Earth in the TETG. Theory of a Screening Experiment of a Sample Body at the Equator Using the Earth as a Screen. Nuovo Cimento, C5, 189.

[27] Jaakkola, T. (1996) Action-at-a-Distance and Local Action in Gravitation: Discus- 
sion and Possible Solution of the Dilemma. Apeiron, 3, 61-75.

[28] Van Flandern, T. (1999) Dark Matter, Missing Planets and New Comets. 2nd Edition, North Atlantic Books, Berkeley, 2-4.

[29] Edwards, M.R. (2002) Pushing Gravity: New Perspectives on Le Sage's Theory of Gravitation. C. Roy Keys Inc., Montreal.

[30] Edwards, M.R. (2007) Photon-Graviton Recycling as Cause of Gravitation. Apeiron, $14,214$.

[31] Corda, C. (2009) Interferometric Detection of Gravitational Waves: The Definitive Test for General Relativity. International Journal of Modern Physics D, 18, 2275. https://doi.org/10.1142/S0218271809015904

[32] Lev, F.M. (2010) Is Gravity an Interaction? Physics Essays, 23, 355.

[33] Riemann, B. (1854) On the Hypotheses Which Lie at the Bases of Geometry. Translated by William Kingdon Clifford. Nature, Vol. VIII, Nos. 183, 184, pp. 14-17, 36, 37.

[34] Heaviside, O. (1893) A Gravitational and Electromagnetic Analogy. The Electrician, 31,81 .

[35] Hoyle, F. and Narlikar, J.V. (1964) A New Theory of Gravitation. Proceedings of the Royal Society of London A, 282, 178. https://doi.org/10.1098/rspa.1964.0227

[36] Dirac, P.A.M. (1974) Cosmological Models and the Large Numbers Hypothesis. Proceedings of the Royal Society of London A, 338, 439. https://doi.org/10.1098/rspa.1974.0095

[37] Pereira, M. (2007) Hypergeometrical Universe. In: Smarandache, F. and Christianto, V., Eds., Quantization in Astrophysics, Brownian Motion and Supersymmetry, MathTiger, Chennai, Tamil Nadu, 391-432.

[38] Pereira, M. (2007) The Hypergeometrical Standard Model. In: Smarandache, F. and Christianto, V., Eds., Hadron Models and Related New Energy Issues, InfoLearnQuest Publisher, USA, 382-435.

[39] Ahmadi, M., et al. (2018) Characterization of the 1S-2S Transition in Antihydrogen. Nature, 557, 71-75.

[40] Boehm, C., Fayet, P. and Silk, J. (2003) Light and Heavy Dark Matter Particles. arXiv:0311143.

[41] Arrenberg, S., et al. (2013) Complementarity of Dark Matter Experiments. http://www-public.slac.stanford.edu/snowmass2013/docs/CosmicFrontier/Comple mentarity-27.pdf

[42] Heeck, J. and Zhang, H. (2013) Exotic Charges, Multicomponent Dark Matter and Light Sterile Neutrinos. Journal of High Energy Physics, 2013, 164. https://doi.org/10.1007/JHEP05(2013)164

[43] Aoki, M., et al. (2012) Multi-Component Dark Matter Systems and Their Observation Prospects. arXiv: 1207.3318.

[44] Kusenko, A., Loewenstein, M. and Yanagida, T. (2013) Moduli Dark Matter and the Search for its Decay Line Using Suzaku X-Ray Telescope. Physical Review D, 87, Article ID: 043508. https://doi.org/10.1103/PhysRevD.87.043508

[45] Feldman, D., Liu, Z., Nath, P. and Peim, G. (2010) Multicomponent Dark Matter in Supersymmetric Hidden Sector Extensions. Physical Review D, 81, Article ID: 095017. https://doi.org/10.1103/PhysRevD.81.095017

[46] Feng, J.L. (2010) Dark Matter Candidates from Particle Physics and Methods of Detection. Annual Review of Astronomy and Astrophysics, 48, 495-545. 
https://doi.org/10.1146/annurev-astro-082708-101659

[47] Zurek, K.M. (2009) Multi-Component Dark Matter. arXiv: 0811.4429.

[48] Spolyar, D., Freese, K. and Gondolo, P. (2007) Dark Matter and the First Stars: A New Phase of Stellar Evolution. arXiv:0705.0521.

[49] Ripamonti, E. and Abel, T. (2005) The Formation of Primordial Luminous Objects. arXiv:0507130.

[50] Lee, B.W. and Weinberg, S. (1977) Cosmological Lower Bound on Heavy-Neutrino Masses. Physical Review Letters, 39, 165. https://doi.org/10.1103/PhysRevLett.39.165

[51] Dicus, D.A., Kolb, E.W. and Teplitz, V.L. (1977) Cosmological Upper Bound on Heavy-Neutrino Lifetimes. Physical Review Letters, 39, 168. https://doi.org/10.1103/PhysRevLett.39.168

[52] Dicus, D.A., Kolb, E.W. and Teplitz, V.L. (1978) Cosmological Implications of Massive, Unstable Neutrinos. Astrophysical Journal, 221, 327-341. https://doi.org/10.1086/156031

[53] Gunn, J.E., et al. (1978) Some Astrophysical Consequences of the Existence of a Heavy Stable Neutral Lepton. Astrophysical Journal, 223, 1015-1031. https://doi.org/10.1086/156335

[54] Stecker, F.W. (1978) The Cosmic Gamma-Ray Background from the Annihilation of Primordial Stable Neutral Heavy Leptons. Astrophysical Journal, 223, 1032-1036. https://doi.org/10.1086/156336

[55] Zeldovich, Ya.B., Klypin, A.A., Khlopov, M.Yu. and Chechetkin, V.M. (1980) Astrophysical Constraints on the Mass of Heavy Stable Neutral Leptons. Soviet Journal of Nuclear Physics, 31, 664.

[56] García, R., et al. (2007). Tracking Solar Gravity Modes: The Dynamics of the Solar Core. Science, 316, 1591-1593. https://doi.org/10.1126/science.1140598

[57] Fossat, E., et al. (2017) Asymptotic $g$ Modes: Evidence for a Rapid Rotation of the Solar Core. Astronomy \& Astrophysics, 604, Article No. A40. https://doi.org/10.1051/0004-6361/201730460

[58] Zhang, J., et al. (2005) Inner Core Differential Motion Confirmed by Earthquake Waveform Doublets. Science, 309, 1357-1360. https://doi.org/10.1126/science.1113193

[59] Guillot, T., et al. (2018) A Suppression of Differential Rotation in Jupiter's Deep Interior. Nature, 555, 227-230. https://doi.org/10.1038/nature25775

[60] Cole, G.H.A. and Woolfson, M.M. (2002) Planetary Science: The Science of Planets around Stars. Institute of Physics Publishing, 36-37, 380-382. https://doi.org/10.1887/075030815X

[61] Kinver, M. (2009) Global Average Temperature May Hit Record Level in 2010. BBC. Retrieved 22 April 2010.

[62] Alsop, J.W. (1934) Beam to Kill Army at 200 Miles, Tesla's Claim on 78th Birthday. The New York Herald Tribune. https://en.wikisource.org/wiki/The_New_York_Herald_Tribune/1934/07/11/Beam to_Kill_Army_at_200_Miles,_Tesla\%27s_Claim_on_78th_Birthday

[63] Dirac, P.A.M. (1937) The Cosmological Constants. Nature, 139, 323. https://doi.org/10.1038/139323a0

[64] Pontecorvo, B. and Smorodinsky, Y. (1962) The Neutrino and the Density of Matter 
in the Universe. Soviet Physics-JETP, 14, 173-176.

[65] Kajita, T. (1999) Atmospheric Neutrino Results from Super-Kamiokande and Kamiokande-Evidence for $v_{\mu}$ Oscillations. Nuclear Physics B-Proceedings Supplements, 77, 123-132.

[66] McDonald, A.B. (2003) Neutrino Properties from Measurements Using Astrophysical and Terrestrial Sources. arXiv:0310775.

[67] Sakharov, A.D. (1968) Vacuum Quantum Fluctuations in Curved Space and the Theory of Gravitation. Soviet Physics Doklady, 12, 1040.

[68] Visser, M. (2002) Sakharov's Induced Gravity: A Modern Perspective. Modern Physics Letters A, 17, 977. https://doi.org/10.1142/S0217732302006886

[69] Barcelo, C., Liberati, S. and Visser, M. (2011) Analogue Gravity. Living Reviews in Relativity, 14, 3. https://doi.org/10.12942//rr-2011-3

[70] Dirac, P.A.M. (1931) Quantized Singularities in the Electromagnetic Field. Proceedings of the Royal Society of London A, 133, 60.

http://users.physik.fu-berlin.de/ kleinert/files/dirac1931.pdf https://doi.org/10.1098/rspa.1931.0130

[71] Harari, H. (1979) A Schematic Model of Quarks and Leptons. Physics Letters B, 86, 83-86. https://doi.org/10.1016/0370-2693(79)90626-9

[72] Shupe, M.A. (1979) A Composite Model of Leptons and Quarks. Physics Letters B, 86, 87-92.

[73] D’Souza, I.A. and Kalman, C.S. (1992) Preons: Models of Leptons, Quarks and Gauge Bosons as Composite Objects. World Scientific, Singapore. https://doi.org/10.1142/1700

[74] Thompson, D.J. (2003) Gamma Ray Pulsars: Multiwavelength Observations. arXiv:0312272.

[75] Ansoldi, S., et al. (2015) Teraelectronvolt Pulsed Emission from the Crab Pulsar Detected by MAGIC. arXiv:1510.07048.

[76] Chen, G., et al. (2015) NuSTAR Observations of the Young, Energetic Radio Pulsar PSR B1509-58. arXiv:1507.08977.

[77] Ackermann, M., et al. (2013) High-Energy Gamma-Ray Emission from Solar Flares: Summary of Fermi LAT Detections and Analysis of Two M-Class Flares. arXiv:1304.3749.

[78] Bjerknes, V.F.K. (1906) Fields of Force. Columbia University Press, New York.

[79] Mansuripur, M. (2012) Nature of Electric and Magnetic Dipoles Gleaned from the Poynting Theorem and the Lorentz Force Law of Classical Electrodynamics. arXiv:1208.0873.

[80] Michon, G.P. Electromagnetism: Maxwell's Equations and Their Solutions-Numericana. http://www.numericana.com/answer/maxwell.htm

[81] Brown, K.S. Magnetic Dipoles. http://www.mathpages.com/home/kmath694/kmath694.htm

[82] Harmuth, H.F. and Lukin, K.A. (2000) Interstellar Propagation of Electromagnetic Signals. Kluwer Academic/Plenum Publishers, New York. https://doi.org/10.1007/978-1-4615-4247-6

[83] Harmuth, H.F. and Lukin, K.A. (2002) Propagation of Short Electromagnetic Pulses through Nonconducting Media with Electric and Magnetic Dipole Currents. Radio Physics and Radio Astronomy, 7, 362. 
[84] Beck, R. and Wielebinski, R. (2013) Magnetic Fields in Galaxies. In: Oswalt, T.D. and Gilmore, G., Eds., Planets, Stars and Stellar Systems, Springer, Dordrecht, 641-723. https://doi.org/10.1007/978-94-007-5612-0_13

[85] Han, J.L. (2003) The Large-Scale Magnetic Field Structure of Our Galaxy: Efficiently Deduced from Pulsar Rotation Measures. In: Uyaniker, B., Reich, W. and Wielebinski, R., Eds., The Magnetized Interstellar Medium, Copernicus GmbH, Katlenburg-Lindau, 3-12.

[86] Pitkanen, M. (2015) “Invisible Magnetic Fields” as Dark Magnetic Fields. TGD Diary. http://matpitka.blogspot.com/2015/09/invisible-magnetic-fields-as-dark.html?m=0

[87] Bennett, C.L., et al. (2013) Nine-Year Wilkinson Microwave Anisotropy Probe (WMAP) Observations: Final Maps and Results. arXiv:1212.5225.

[88] Fixsen, D.J. (2009) The Temperature of the Cosmic Microwave Background. The Astrophysical Journal, 707, 916-920. https://doi.org/10.1088/0004-637X/707/2/916

[89] Fixsen, D.J., et al. (1996) The Cosmic Microwave Background Spectrum from the Full COBE FIRAS Data Set. The Astrophysical Journal, 473, 576-587. https://doi.org/10.1086/178173

[90] Finkbeiner, D.P., Davis, M. and Schlegel, D.J. (1999) Extrapolation of Galactic Dust Emission at 100 Microns to CMBR Frequencies Using FIRAS. arXiv:9905128.

[91] Draine, B.T. and Lazarian, A. (1998) Electric Dipole Radiation from Spinning Dust Grains. The Astrophysical Journal, 508, 157-179. https://doi.org/10.1086/306387

[92] Finkbeiner, D.P. and Schlegel, D.J. (1999) Interstellar Dust Emission as a CMBR Foreground. arXiv: 9907307.

[93] Lagache, G., et al. (1999) First Detection of the Warm Ionized Medium Dust Emission. Implication for the Cosmic Far-Infrared Background. arXiv:9901059.

[94] Finkbeiner, D.P., Davis, M. and Schlegel, D.J. (2000) Detection of a Far IR Excess with DIRBE at 60 and 100 Microns. arXiv:0004175.

[95] Siegel, P.H. (2002) Terahertz Technology. IEEE Transactions on Microwave Theory and Techniques, 50, 910-928. https://doi.org/10.1109/22.989974

[96] Phillips, T.G. and Keene, J. (1992) Submillimeter Astronomy (Heterodyne Spectroscopy). Proceedings of the IEEE, 80, 1662-1678. https://doi.org/10.1109/5.175248

[97] Dupac, X., et al. (2003) The Complete Submillimeter Spectrum of NGC 891. arXiv:0305230.

[98] Aguirre, J.E., et al. (2003) The Spectrum of Integrated Millimeter Flux of the Magellanic Clouds and 30-Doradus from TopHat and DIRBE Data. arXiv:0306425.

[99] Pope, A., et al. (2006) Using Spitzer to Probe the Nature of Submillimetre Galaxies in GOODS-N. arXiv: 0603409.

[100] Marshall, J.A., et al. (2007) Decomposing Dusty Galaxies. I. Multi-Component Spectral Energy Distribution Fitting. arXiv:0707.2962.

[101] Zyga, L. (2015) Why Do Measurements of the Gravitational Constant Vary So Much? Phys.org. https://phys.org/news/2015-04-gravitational-constant-vary.html

[102] Gough, D.O. (1981) Solar Interior Structure and Luminosity Variations. Solar Physics, 74, 21-34. https://doi.org/10.1007/BF00151270

[103] Stanimirovic, S., Altschuler, D., Goldsmith, P. and Salter, C. (2002) Single-Dish Radio Astronomy: Techniques and Applications. ASP Conference Proceedings, 278. Astronomical Society of the Pacific, San Francisco, 251-269.

[104] Lorimer, D.R., and Kramer, M. (2005) Handbook of Pulsar Astronomy. In: Cam- 
bridge Observing Handbooks for Research Astronomers, Vol. 4, Cambridge University Press, Cambridge, New York.

[105] Keane, E.F., et al. (2016) A Fast Radio Burst Host Galaxy. Nature, 530, 453-456. https://doi.org/10.1038/nature17140 